\title{
Imaging of angiogenesis
}

\author{
Willem J. M. Mulder · Arjan W. Griffioen
}

Published online: 18 June 2010

(c) The Author(s) 2010. This article is published with open access at Springerlink.com

Imaging of angiogenesis is an important tool in the diagnosis of disease. Small molecule or nanoparticular probes are currently developed to identify angiogenic endothelium with non-invasive imaging techniques such as magnetic resonance and ultrasound imaging. These techniques are very valuable since they would not only contribute to improved diagnosis, but will also allow monitoring of angiogenesis intervention therapies, early detection of disease, as well therapy development. Such techniques will ultimately also contribute to the introduction of personalized medicine. The current special issue of Angiogenesis is put together to highlight several interesting and new developments in this field.

Intervention in angiogenesis has currently made its way into daily clinical practice. Several angiogenesis inhibitors have been approved by the Food and Drug Administration (FDA) for therapeutic use in the therapy of disease, most commonly in oncological pathologies. Since angiogenesis is a multidisciplinary theme, application of angiogenesis inhibition is also expected for other angiogenic diseases such as rheumatoid arthritis, atherosclerosis, endometriosis and psoriasis [1]. Clinical application has been approved for the therapy of many cancer types and eye diseases, such as age-related macular degeneration. In the field of cancer, neutralization of the key angiogenic growth factor VEGF is

W. J. M. Mulder $(\square)$

Translational and Molecular Imaging Institute, Mount Sinai School of Medicine, One Gustave L. Levy Place, Box 1234, New York, NY, USA

e-mail: Willem.Mulder@mountsinai.org

\section{A. W. Griffioen}

Angiogenesis Laboratory, Department of Medical Oncology, VUmc-Cancer Center Amsterdam, VU University Medical Center, Amsterdam, The Netherlands daily clinical practice, mostly in combination with chemotherapeutic agents. While the first approved agent, Avastin/bevacizumab, is an anti-VEGF antibody based therapeutic, later generation drugs are collectively based on inhibition of VEGF (and other growth factor) signaling by inhibition of tyrosine kinase inhibitors. This type of inhibitors is now widely tested and clinical studies have led to FDA approval for several of them. Application is possible now for many tumor types and is especially successful in renal cell cancer, where monotherapy with Sutent/sunitinib is nowadays first line therapy.

While these examples picture the exploitation of angiogenesis for development of therapy, there is also an active field of science exploiting angiogenesis or angiogenesis inhibitors for diagnostic purposes making use of imaging technologies. Imaging of diseased tissue by exploiting vascular permeability or vascular targeting is a promising tool in modern medicine. The latter approach depends on identification of targets that are upregulated at angiogenic blood vessels as compared to quiescent normal blood vessels and on the availability of probes that bind selectively and with high affinity to such targets. Different genomic screening approaches, performed worldwide, identified markers of angiogenic endothelial cells. Among these markers are molecules such as $\alpha_{\mathrm{v}} \beta_{3}$ integrin, CD13, vimentin, VEGF receptor, and galectin-1, which have shown promise in imaging of angiogenesis [2-4].

The field of angiogenesis research has been initiated by the hypothesis that the growth of tumors is dependent on the formation of new blood vessels, put forward by Folkman [5] in the early 1970s. This idea indicated that angiogenesis inhibitors might be discovered and employed as therapy against angiogenic diseases. The process of angiogenesis is an intricately regulated cascade of processes that occurs in growing tissues where, for example, 
conditions of hypoxia have turned on the production of angiogenic growth factors such as the families of vascular endothelial cell growth factors (VEGFs) and fibroblast growth factors (FGFs). Preexisting endothelial cells in capillaries can sense that and subsequently produce proteases to dissolve the basement membrane and extracellular matrix. Thereby endothelial cells migrate into the direction of the stimulus. Endothelial cells subsequently proliferate and form new vascular sprouts that become functional blood vessels after the attraction of accessory cells such as pericytes and the formation of a new rigid extracellular matrix [6]. This angiogenesis cascade provides opportunities for intervention in every single step separately, and inhibitors for each of these steps have been discovered and are being developed in clinical studies. Anti-angiogenesis compounds can specifically and directly inhibit the proliferation of endothelial cells (TNP-470/caplostatin, platelet factor-4) or interfere directly with the migratory activity of these cells (endostatin, integrin antagonists). Alternatively, they can inhibit the production or activity of metalloproteinases (MMPs), inducing a hampered mobility of endothelial cells. However, the best developed angiogenesis inhibitors are the ones that act indirectly, either by clearing angiogenic growth factors from the circulation, blocking the corresponding growth factor receptors, or by intervention in the intracellular signaling pathways activated by these growth factors.

A variety of imaging modalities is available to visualize and characterize the angiogenic vasculature [7]. In a clinical setting these include magnetic resonance imaging (MRI), positron emission tomography (PET), single photon emission computed tomography (SPECT), ultrasound imaging and computed tomography (CT) [8]. Preclinically, all the aforementioned imaging modalities are available, but usually as dedicated small animal scanners with a smaller field of view and higher spatial resolution [9]. In addition, optical in vivo imaging techniques such as intravital microscopy, near infrared fluorescence (NIRF) and bioluminescence imaging are frequently employed to evaluate angiogenesis in small laboratory animals [10].

Dynamic contrast enhanced (DCE) MRI is one of the most widely and commonly used imaging methods to visualize tumor angiogenesis in cancer patients and to evaluate angiostatic therapies [11]. The application of contrast enhanced CT imaging is also being explored for perfusion imaging of cancers in patients. Both DCE MRI and CT provide information about the vascular permeability of tumors, but do not directly visualize the newly formed vasculature. Fluorodeoxyglucose (FDG) PET imaging allows quantitative imaging of glucose metabolism. Since tumor glucose metabolism is expected to decrease when nutrient vessels regress after the onset of angiostatic therapy, as an indirect marker for angiogenesis, a decrease in glucose metabolism may be visualized by FDG-PET. Unfortunately, changes in vascular permeability and FDG uptake may not necessarily be the result of changes in ongoing angiogenesis, but can also be the result of a variety of different processes, including hypoxia or the collapse of the tumor microvasculature. More recently, contrast enhanced MRI and CT as well as FDG-PET have been explored for the study of neovascularization in atherosclerosis in a number of studies [12,13]. Although in its infancy, these studies have shown a clear correlation between atherosclerotic plaque permeability, microvessel density and vulnerability. Interestingly, Calcagno et al. [12], who also contributed to this special issue, have shown parameters obtained in vivo with DCE-MRI and FDG-PET to correlate with the plaque microvessel density determined ex vivo, in a rabbit model of atherosclerosis.

To enable a more specific evaluation of angiogenesis, target-specific imaging methods have been developed for PET and SPECT imaging, MRI, ultrasound as well as optical imaging [14]. Such molecular imaging methods exploit probes that, after intravenous administration, specifically target molecular epitopes upregulated at the angiogenic vasculature. These probes are labeled with tracers and/or materials that allow their visualization with one (or more) of the aforementioned imaging techniques. The nuclear imaging techniques (PET and SPECT) inherently rely on target-specific probes. To that end, targeting ligands such as antibodies, proteins and peptides are labeled with radioactive isotopes. Most notably, the $\alpha_{v} \beta_{3}$ integrin specific RGD peptide has been studied and applied in both preclinical and clinical studies, which has resulted in the development of a wide variety of radiolabeled RGD analogs [15]. In addition, different other endothelial cell markers have been exploited to image angiogenesis by PET and SPECT, including the VEGF receptor or adhesion molecules [16].

The development of nanoparticulate molecular imaging probes has shown great progress in the past decade [17]. Such probes, carrying a high payload of contrast generating materials, have shown to be especially useful for MR molecular imaging of angiogenesis, since MRI is a relatively insensitive technique that requires $\mu \mathrm{M}$ concentrations of contrast agents to accumulate to allow their visualization [18]. For optical techniques, quantum dots have shown great potential because these semiconductor nanoparticles exhibit some unique properties that make them resistant to photobleaching and provide a very narrow and tunable excitation wavelength [19]. Interestingly, nanoparticles allow the integration of multiple materials and agents for multimodal imaging purposes and/or the combination of target-specific therapy [20].

For the current special issue on "Imaging of Angiogenesis" we have invited a series of renowned expert 
investigators to contribute a state-of-the-art review. We attempted to put together an issue that covers the abovementioned wide range of applications in this field. The issue starts with a contribution, which also provided the cover art, about vascular permeability and lymphatic drainage imaging in experimental cancer by Vandoorne, Addadi and Neeman [21]. Calcagno et al. [12] [22] show the potential of contrast enhanced MR imaging to identify the vulnerability of atherosclerotic plaques'. MRI of angiogenesis in the brain as a result of stroke has been reviewed by investigators of the NRM group of Dijkhuizen in Utrecht, The Netherlands [23]. A contribution from a Norwegian group by Hak et al. [24] exemplifies the use of window chamber models to visualize nanoparticle targeting in tumors by intravital microscopy. We have contributed a mini review about paramagnetic quantum dots for multimodal of tumor angiogenesis [25], while Snoeks, Löwik and Kaijzel from the Leiden University Medical Center in The Netherlands highlight the latest developments in optical approaches to image and characterize angiogenesis [26]. Investigators Jansen, Koutcher and Shukla-Dave at Memorial Sloan-Kettering Cancer Center [27] reviewed the field of head and neck squamous cell carcinoma imaging. The Biomedical NMR group of the Eindhoven University of Technology (Nicolay, Strijkers et al.) summarized their work on multimodal lipoomes [28], while Willmann et al. from Stanford University School of Medicine wrote an overview of their ultrasound based tumor angiogenesis imaging work [29]. The special issue is wrapped up with a great contribution by Lanza et al. [20] from Washington University of Medicine. They give a summary of their work with angiogenesis specific perfluorocarbon nanoparticles for molecular MRI and therapy of cancer and atherosclerosis [30].

We are confident that this special issue of Angiogenesis is a valuable documentation of the current technologies available to image neovaculature formation.

Open Access This article is distributed under the terms of the Creative Commons Attribution Noncommercial License which permits any noncommercial use, distribution, and reproduction in any medium, provided the original author(s) and source are credited.

\section{References}

1. Carmeliet P (2005) Angiogenesis in life, disease and medicine. Nature 438:932-936

2. Brooks PC, Clark RA, Cheresh DA (1994) Requirement of vascular integrin alpha v beta 3 for angiogenesis. Science 264:569-571

3. Thijssen VL, Postel R, Brandwijk RJ, Dings RP, Nesmelova I, Satijn S, Verhofstad N, Nakabeppu Y, Baum LG, Bakkers J, Mayo KH, Poirier F, Griffioen AW (2006) Galectin-1 is essential in tumor angiogenesis and is a target for antiangiogenesis therapy. Proc Natl Acad Sci USA 103:15975-15980
4. van Beijnum JR, Dings RP, van der LE, Zwaans BM, Ramaekers FC, Mayo KH, Griffioen AW (2006) Gene expression of tumor angiogenesis dissected: specific targeting of colon cancer angiogenic vasculature. Blood 108:2339-2348

5. Folkman J (1971) Tumor angiogenesis: therapeutic implications. N Engl J Med 285:1182-1186

6. Griffioen AW, Molema G (2000) Angiogenesis: potentials for pharmacologic intervention in the treatment of cancer, cardiovascular diseases, and chronic inflammation. Pharmacol Rev $52: 237-268$

7. McDonald DM, Choyke PL (2003) Imaging of angiogenesis: from microscope to clinic. Nat Med 9:713-725

8. Jaffer FA, Weissleder R (2005) Molecular imaging in the clinical arena. JAMA 293:855-862

9. Rudin M, Weissleder R (2003) Molecular imaging in drug discovery and development. Nat Rev Drug Discov 2:123-131

10. Shah K, Weissleder R (2005) Molecular optical imaging: applications leading to the development of present day therapeutics. NeuroRx 2:215-225

11. Padhani AR (2002) Dynamic contrast-enhanced MRI in clinical oncology: current status and future directions. J Magn Reson Imaging 16:407-422

12. Calcagno C, Cornily JC, Hyafil F, Rudd JH, Briley-Saebo KC, Mani V, Goldschlager G, Machac J, Fuster V, Fayad ZA (2008) Detection of neovessels in atherosclerotic plaques of rabbits using dynamic contrast enhanced MRI and 18F-FDG PET. Arterioscler Thromb Vasc Biol 28:1311-1317

13. Hyafil F, Cornily JC, Feig JE, Gordon R, Vucic E, Amirbekian V, Fisher EA, Fuster V, Feldman LJ, Fayad ZA (2007) Noninvasive detection of macrophages using a nanoparticulate contrast agent for computed tomography. Nat Med 13:636-641

14. Weissleder R, Mahmood U (2001) Molecular imaging. Radiology 219:316-333

15. Haubner R, Decristoforo C (2009) Radiolabelled RGD peptides and peptidomimetics for tumour targeting. Front Biosci 14:872886

16. Cai W, Chen X (2008) Multimodality molecular imaging of tumor angiogenesis. J Nucl Med 49(Suppl 2):113S-128S

17. Cormode DP, Skajaa T, Fayad ZA, Mulder WJ (2009) Nanotechnology in medical imaging: probe design and applications. Arterioscler Thromb Vasc Biol 29(7):992-1000

18. Mulder WJ, Strijkers GJ, van Tilborg GA, Griffioen AW, Nicolay K (2006) Lipid-based nanoparticles for contrast-enhanced MRI and molecular imaging. NMR Biomed 19:142-164

19. Medintz IL, Uyeda HT, Goldman ER, Mattoussi H (2005) Quantum dot bioconjugates for imaging, labelling and sensing. Nat Mater 4:435-446

20. Lanza GM, Winter PM, Caruthers SD, Hughes MS, Cyrus T, Marsh JN, Neubauer AM, Partlow KC, Wickline SA (2006) Nanomedicine opportunities for cardiovascular disease with perfluorocarbon nanoparticles. Nanomedicine 1:321-329

21. Vandoorne K, Addadi Y, Neeman M (2010) Visualizing vascular permeability and lymphatic drainage using labeled serum albumin. Angiogenesis. doi: 10.1007/s10456-010-9170-4

22. Calcagno C, Mani V, Ramachandran S, Fayad ZA (2010) Dynamic contrast enhanced (DCE) magnetic resonance imaging (MRI) of atherosclerotic plaque angiogenesis. Angiogenesis. doi: 10.1007/s10456-010-9172-2

23. Seevinck PR, Deddens LH, Dijkhuizen RM (2010) Magnetic resonance imaging of brain angiogenesis after stroke. Angiogenesis. doi: 10.1007/s10456-010-9174-0

24. Hak S, Reitan N, Haraldseth O, de Lange Davies C (2010) Intravital microscopy in window chambers: a unique tool to study tumor angiogenesis and delivery of nanoparticles. Angiogenesis. doi: 10.1007/s10456-010-9176-y 
25. Mulder WJM, Strijkers GJ, Nicolay K, Griffioen AW (2010) Quantum dots for multimodal molecular imaging of angiogenesis. Angiogenesis. doi: 10.1007/s10456-010-9177-x

26. Snoeks TJA, Löwik CWGM, Kaijzel EL (2010) 'In vivo' optical approaches to angiogenesis imaging. Angiogenesis. doi: 10.1007/ s10456-010-9168-y

27. Jansen JFA, Koutcher JA, Shukla-Dave A (2010) Non-invasive imaging of angiogenesis in head and neck squamous cell carcinoma. Angiogenesis. doi: 10.1007/s10456-010-9167-z

28. Strijkers GJ, Kluza E, Van Tilborg GAF, van der Schaft DWJ, Griffioen AW, Mulder WJM, Nicolay K (2010) Paramagnetic and fluorescent liposomes for target-specific imaging and therapy of tumor angiogenesis. Angiogenesis. doi: 10.1007/s10456-010-9165-1

29. Deshpande N, Pysz MA, Willmann JK (2010) Molecular ultrasound assessment of tumor angiogenesis. Angiogenesis. doi: 10.1007/s10456-010-9175-z

30. Lanza GM, Winter PM, Caruthers SD, Hughes MS, Hu G, Schmieder AH, Wickline SA (2010) Theragnostics for tumor and plaque angiogenesis with perfluorocarbon nanoemulsions. Angiogenesis. doi: 10.1007/s10456-010-9166-0 